\title{
Staging laparoscopy for locally advanced gastric cancer in Chinese patients: a multicenter prospective registry study
}

\author{
Ziyu Li ${ }^{1}$, Zhemin Li ${ }^{1}$, Lianhai Zhang ${ }^{1}$, Qian Liu², Zhenjun Wang ${ }^{3}$, Zhongtao Zhang ${ }^{4}$, Gang Xiao ${ }^{5}$, Wei Fu ${ }^{6}$,
} Xin Wang ${ }^{7}$, Yingjiang Ye ${ }^{8}$, Jianchun $\mathrm{Yu}^{9}$, Fei $\mathrm{Li}^{10}$, Lin Chen ${ }^{11}$, Shibin Wang ${ }^{12}$ and Jiafu Ji, ${ }^{13^{*}}$ (D

\begin{abstract}
Background: Staging laparoscopy(SL) is a recommended technique for the staging of Gastric Cancer(GC) and provides the indication for a radical surgery. Considering the medical practice in China, the standardized and regular usage of SL is yet to be spread. However, existing guidelines vary and make an ambiguity of indication for SL. Besides, the specific indication for Chinese patients remains a niche. This study aims to the essential, missing information of Chinese patients and tries to normalize the indication of LS in medical practice in China.

Methods: The study is a prospective, multicenter cohort study being conducted in China with a total of 450 patients, all diagnosed with locally advanced gastric cancer (cT2-4 NO-3 MO, no evidence of intra-abdominal dissemination) through Computed Tomography(CT) and/or Endoscopic Ultrasonography(EUS). Peritoneal lavage is regularly performed during the SL. Multivariate Cox regression model and receiver-operator characteristic(ROC) analysis will be used to analyze the significant risk factors of intra-abdominal metastasis(including peritoneal dissemination and a positive cytological result).

Discussion: This confirmatory study will provide us with the specific positive rate of intraabdominal metastasis of GC in China, compared with empirical evidence of $20 \%$. We expect this trial will contribute to our discovery of the specific risk factors of intra-abdominal metastasis of Chinese patients and to the stimulating and performing of minimally invasive surgical procedures.
\end{abstract}

Trial registration: ClinicalTrials.gov: registration number NCT02172690.

Keywords: Gastric cancer, Laparoscopy, Peritoneal metastasis, Cytology

\section{Background}

Gastric cancer is a major cause of cancer death worldwide with a 40 percentage of cases occurring in China [1]. Individualized treatment based upon tumor stage takes on a widely accepted strategy, and surgery remains the most effective treatment modality.

Most of the Chinese patients are diagnosed with locally advanced stage, suffering the substantial intra-abdominal

\footnotetext{
* Correspondence: jiafuj@hotmail.com

${ }^{1}$ Key laboratory of Carcinogenesis and Translational Research (Ministry of Education), Department of Gastrointestinal Surgery, Peking University Cancer Hospital and Institute, Beijing 100142, China

${ }^{13}$ Key laboratory of Carcinogenesis and Translational Research (Ministry of Education), Department of Gastrointestinal Surgery, Peking University Cancer Hospital and Institute, Beijing 100142, China

Full list of author information is available at the end of the article
}

metastases with higher risks. Precise preoperative staging is particularly important for therapeutic strategies and prognostic evaluation.

Precise staging is a foremost method that benefits the successful surgical intervention of cancer, contributed to the improvement of medical tools: despite the fact that preoperative imaging techniques have significantly improved the diagnostic accuracy of wall infiltration and lymph node involvement, the detection of peritoneal dissemination still needs to be developed; positive intraperitoneal findings of free cancer cells has been proved to be related with poor survival yet could only be confirmed during surgical procedure $[2,3]$.

SL has been suggested useful in detecting unrecognized peritoneal dissemination, and in collecting peritoneal lavage 
fluid for cytological examination with minimal invasion. However, indication for SL remains controversial. NCCN guideline recommends an indication for SL of T3 and/or $\mathrm{N}+$; ESMO, IB-III; JGCA, none, merely mentioned SL only on the condition of neoadjuvant Chemoradiotherapy [4-6]. No specific recommended indications for Chinese patients can be referred to for lack of solid evidence.

The aim of this study is to acquire a basic data on the positive rate of SL in Chinese patients and to identify the risk factors associated with intra-abdominal metastasis.

\section{Methods}

\section{The design of study}

This study evaluates the efficacy of SL with peritoneal cytology, and determine the indications if possible.

Patients who meet the inclusion criteria will be enrolled consecutively, with their preliminary data collected using a web-based case report form. When the whole data-collecting is completed, factors associated with intra-abdominal metastasis will be analyzed by multivariate Cox regression model. Receiver-operator characteristic (ROC) will be used to find out risk factors of intra-abdominal metastasis. Figure 1 shows the CONSORT diagram.

\section{Recruitment}

Patients with pathologically confirmed gastric adenocarcinoma are recruited. If the patient meets all inclusion criteria, following data will be recorded: basic information including age, gender, height, body weight et al., clinical information including CT stage(enhanced CT is mandatory required), EUS stage, result of blood examinations such as tumor marker (CEA, CA19-9, CA72-4, CA12-5), blood cell count, albumin et al. If there been any differences between CT \& EUS, it is required to record them separately.

\section{Staging laparoscopy}

If the patient is confirmed at stage cT2-4bN0-3 M0, which means no sign of distant metastasis detected by
CT/EUS, he/she undergoes SL. Following data will be recorded: intraoperative finding including the peritoneal dissemination, and the peritoneal lavage; video of surgery and pictures demonstrating the extent of peritoneal dissemination is required.

\section{Follow-up}

Postoperative hospital stay and complications will be recorded, the follow-up time will be 30 days after surgery. Only those who undergoes DL alone will be included into safety analysis since the complications will be confused if the patient undergo radical surgery immediately after SL.

\section{Study endpoint}

The primary target of this study is to obtain the result of SL. Either peritoneal dissemination or a positive result of cytological examination will be recorded as a positive result. If the pathological or cytological examination describes "suspicious", the result will be characterized as positive, consistent with our current practice.

The secondary aim is to evaluate the safety of SL.

\section{Selection of candidate patients}

1) Age $\geq 18$;

2) Histological confirmed diagnosis of primary gastric carcinoma (including esophagus-gastric junction).

3) Karnofsky score $\geq 70$;

4) Abdominally enhanced spinal CT and endoscopic ultrasonography (EUS) showed a clinical stage of T2-4bN0-3bM0;

5) Fit for radical gastrectomy or neo-adjuvant chemotherapy;

6) Willing to comply with protocol, and provide informed consent forum.

The exclusion criteria included:

1. Unfit for laparoscopic operation or general anesthesia (suspicious abdominal adhesions, severe cardiopulmonary disease, etc.);

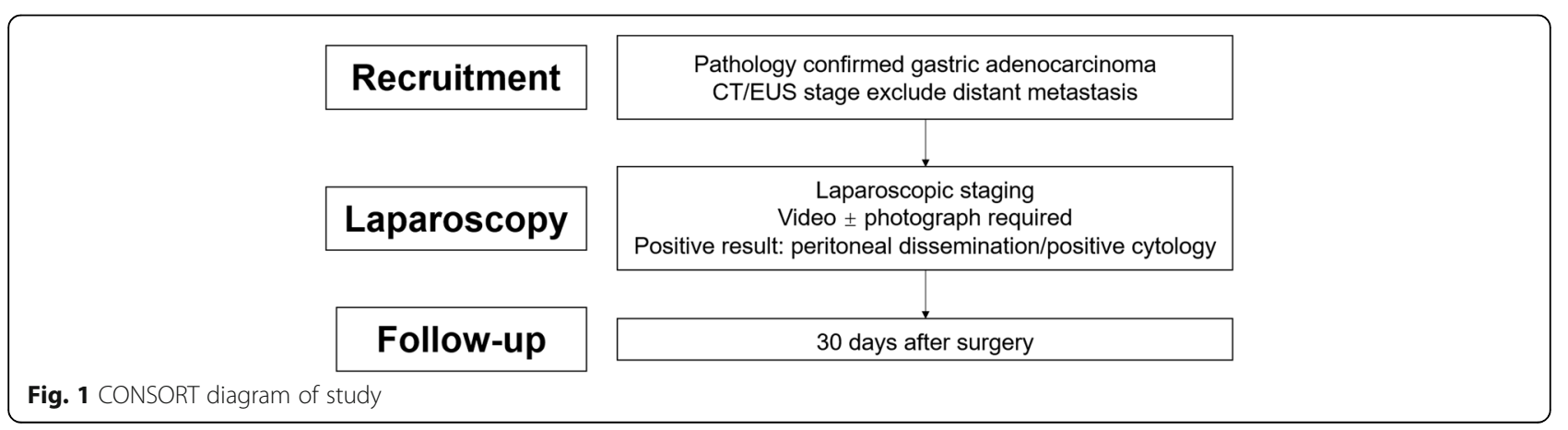


2. Underwent emergency surgery to relieve obstruction, bleeding or perforation.

\section{SL technique}

SL was performed under general anesthesia. The patient is placed in a supine position. A $10-\mathrm{mm}$ disposable trocar (observing hole) is inserted into the sub-umbilicus, and a $30^{\circ}$ telescope is used. Another $10-\mathrm{mm}$ trocar and a 5-mm trocar (operating hole) are inserted into the right upper quadrant and left upper quadrant, respectively. Prior to any manipulation, $250 \mathrm{ml}$ of warm normal saline is infused into subphrenic space, subhepatic space, omentum, bilateral paracolic sulci and the pouch of Douglas. The irrigation should be carefully operated to avoid the primary tumor. At least $100 \mathrm{ml}$ of fluid is aspirated from the subphrenic space, subhepatic space and Douglas' pouch. The fluid is immediately sent for centrifugation and cytological examination and a systematic inspection of the abdominal cavity is performed clockwise from the right quadrant. Any suspicious lesion will be biopsied and sent for frozen pathologic examination.

\section{Sample size}

This study is a single arm OPC (objective performance criteria) design where the primary evaluation index is the positive rate of SL. Based upon literature, expected event rate is $20 \%$, and clinical acceptable rate is above $15 \%$. Type I error as bilateral $5 \%$, dropout rate as $5 \%$, power as $80 \%$, calculated sample size is 450 . The recruitment process is estimated to be completed by December 2016.

\section{Data analysis}

Clinical characteristics of the DL- and DL+ patients are compared using the Independent samples group $t$ test and chi-square test. To determine the risk factors, all variables found to be significant by the univariate analysis are assessed by binary logistic regression analysis (Method: Backward: Wald, probability for stepwise: 0.5 for entry, 1.0 for removal). The ROC curve and AUC analyses are used to determine sensitivity, specificity, and corresponding cut-off value of each factor. All $P$ values $<.05$ are considered statistically significant. All statistical analyses are carried out using the SPSS statistical software package (version 20).

\section{Discussion}

This study is conducted to prospectively evaluate the positive rate of the result of SL based on samples of Chinese patient and try to discover risk factors of intraabdominal metastasis. SL has been recommended by many guidelines yet different indications is suggested. The standardized operational procedure remains controversial for such practice along with possibly unknown bias. Druing the process of information collecting, data will be prospectively recorded using web-based case report forms.

The results of the study will provide the first prospective multi-center database of SL in China, according to our knowledge, which will provide important figure of $40 \%$ of patients of the world.

\section{Abbreviations \\ AUC: Area under curve; CT: Computed Tomography; EUS: Endoscopic ultrasonography; OPC: Objective performance criteria; ROC: Receiver-operator characteristic; SL: Staging laparoscopy \\ Acknowledgements \\ None. \\ Funding \\ This work was supported by grants supporting the research program of early diagnosis, standardized treatment and therapy effect evaluation of gastric cancer (D141100000414004) form Beijing Ministry of Science and \\ Technology.}

\section{Availability of data and materials}

Since this article is study protocol, it's not applicable.

\section{Authors' contributions}

Ziyu $L$ and $J$ J conceived and designed the study, were responsible for the final decision to submit for publication. Zhemin $L$ wrote the first draft of the manuscript. All authors were responsible for patient recruitment, were involved in development, review, and approval of the manuscript. All authors read and approved the final manuscript.

\section{Authors' information \\ None.}

\section{Ethics approval and consent to participate}

This study is conducted in accordance with the "Declaration of Helsinki" and "Ethical Guidelines for Clinical Research", and has been approved by the Institutional Review Boards of each participating institute. Written informed consent is obtained from all patients before enrollment.

IRBs list:

Ethic Committee of Beijing Cancer Hospital (Peking University Cancer Hospital \& Institute).

Ethic Committee of Cancer Hospital and Institute, Chinese Academy of Medical Sciences.

Ethic Committee of Capital Medical University Affiliated Beijing Chao-Yang Hospital.

Ethic Committee of Beijing Friendship Hospital, Capital Medical University.

Ethic Committee of Beijing Hospital of Ministry of Health.

Ethic Committee of Peking University Third Hospital.

Ethic Committee of Peking University First Hospital.

Ethic Committee of Peking University People's Hospital.

Ethic Committee of Peking Union Medical College Hospital, Peking Union Medical College.

Ethic Committee of Xuanwu Hospital, Capital Medical University.

Ethic Committee of the General Hospital of PLA.

Ethic Committee of the First Affiliated Hospital, General Hospital of PLA.

Consent for publication

Not applicable.

Competing interests

No competing interests declared.

\section{Publisher's Note}

Springer Nature remains neutral with regard to jurisdictional claims in published maps and institutional affiliations. 


\section{Author details}

Key laboratory of Carcinogenesis and Translational Research (Ministry of Education), Department of Gastrointestinal Surgery, Peking University Cancer Hospital and Institute, Beijing 100142, China. ${ }^{2}$ Cancer Hospital and Institute, Chinese Academy of Medical Sciences, Beijing 100021, China. ${ }^{3}$ Department of General Surgery, Capital Medical University Affiliated Beijing Chao-Yang Hospital, Beijing 100020, China. ${ }^{4}$ Department of General Surgery, Beijing Friendship Hospital, Capital Medical University, Beijing 100050, China. ${ }^{5}$ Department of General Surgery, Beijing Hospital of Ministry of Health, Beijing 100730, China. ${ }^{6}$ Department of General Surgery, Peking University Third Hospital, Beijing 100191, China. ${ }^{7}$ Department of General Surgery, Peking University First Hospital, Beijing 100034, China. ${ }^{8}$ Department of Gastrointestinal Surgery, Peking University People's Hospital, Beijing 100044 China. ${ }^{9}$ Department of General Surgery, Peking Union Medical College Hospital, Peking Union Medical College, Beijing 100730, China. ${ }^{10}$ Department of General Surgery, Xuanwu Hospital, Capital Medical University, Beijing 100053, China. ${ }^{11}$ Department of General Surgery, The General Hospital of PLA, Beijing 100853, China. ${ }^{12}$ Department of General Surgery, The First Affiliated Hospital, General Hospital of PLA, Beijing 100048, China. ${ }^{13}$ Key laboratory of Carcinogenesis and Translational Research (Ministry of Education), Department of Gastrointestinal Surgery, Peking University Cancer Hospital and Institute, Beijing 100142, China.

Received: 21 May 2016 Accepted: 15 November 2017

Published online: 10 January 2018

\section{References}

1. Ferlay J, et al. Cancer incidence and mortality worldwide: sources, methods and major patterns in GLOBOCAN 2012. Int J Cancer. 2014;136(5):E359-86.

2. Bentrem $\mathrm{D}$, et al. The value of peritoneal cytology as a preoperative predictor in patients with gastric carcinoma undergoing a curative resection. Ann Surg Oncol. 2005;12(5):347-53.

3. La Torre M, et al. Peritoneal wash cytology in gastric carcinoma. Prognostic significance and therapeutic consequences. Eur J Surg Oncol. 2010;36(10):982-6.

4. Ajani JA, et al. Gastric cancer, version 3.2016, NCCN clinical practice guidelines in oncology[J]. J Natl Compr Cancer Netw. 2016;14(10):1286-1312.

5. Waddell T, et al. Gastric cancer: ESMO-ESSO-ESTRO Clinical Practice Guidelines for diagnosis, treatment and follow-up[J]. Ann Oncol. 2013; 24(suppl_6):vi57-vi63.

6. Japanese Gastric Cancer Association. Japanese gastric cancer treatment guidelines 2010 (ver. 3) [J]. Gastric Cancer. 2011;14(2):113-23.

\section{Submit your next manuscript to BioMed Central and we will help you at every step:}

- We accept pre-submission inquiries

- Our selector tool helps you to find the most relevant journal

- We provide round the clock customer support

- Convenient online submission

- Thorough peer review

- Inclusion in PubMed and all major indexing services

- Maximum visibility for your research

Submit your manuscript at www.biomedcentral.com/submit

CBiomed Central 